\title{
State of the Art in Stereoscopic and Autostereoscopic Displays
}

\author{
This overview covers most of the 3-D displays that are in use today and \\ presents recent developments and advances in this field.
}

\author{
By Hakan Urey, Senior Member IEeE, Kishore V. Chellappan, \\ Erdem Erden, Student Member IEEE, and Phil Surman
}

ABSTRACT | Underlying principles of stereoscopic direct-view displays, binocular head-mounted displays, and autostereoscopic direct-view displays are explained and some early work as well as the state of the art in those technologies are reviewed. Stereoscopic displays require eyewear and can be categorized based on the multiplexing scheme as: 1) color multiplexed (old technology but there are some recent developments; low-quality due to color reproduction and crosstalk issues; simple and does not require additional electronics hardware); 2) polarization multiplexed (requires polarized light output and polarization-based passive eyewear; highresolution and high-quality displays available); and 3) time multiplexed (requires faster display hardware and active glasses synchronized with the display; high-resolution commercial products available). Binocular head-mounted displays can readily provide 3-D, virtual images, immersive experience, and more possibilities for interactive displays. However, the bulk of the optics, matching of the left and right ocular images and obtaining a large field of view make the designs quite challenging. Some of the recent developments using unconventional optical relays allow for thin form factors and open up new possibilities. Autostereoscopic displays are very attractive as they do not require any eyewear. There are many possibilities in this category including: two-view (the simplest

Manuscript received April 2, 2010; revised October 4, 2010; accepted November 19, 2010. Date of publication January 28, 2011; date of current version March 18, 2011. This work was supported by the European Commission within FP7 under Grant 215280 HELIUM3D. The work of $\mathrm{H}$. Urey was supported by TUBA-GEBIP Award.

H. Urey is with the Department of Electrical Engineering, Koç University, Istanbul 34450, Turkey (e-mail: hurey@ku.edu.tr).

K. v. Chellappan is with the University of Southern Mississippi, Hattiesburg, MS 39406 USA (e-mail: vckishore@gmail.com).

E. Erden is with the Center for Research and Education in Optics and Lasers (CREOL), University of Central Florida, Orlando, FL 32816 USA (e-mail: rdmrdn@gmail.com).

P. Surman is with the Imaging and Displays Research Group, De Montfort University, Leicester LE1 9BH, U.K. (e-mail: psurman@dmu.ac.uk).

Digital Object Identifier: 10.1109/JPROC.2010.2098351 implementations are with a parallax barrier or a lenticular screen), multiview, head tracked (requires active optics to redirect the rays to a moving viewer), and super multiview (potentially can solve the accommodation-convergence mismatch problem). Earlier 3-D booms did not last long mainly due to the unavailability of enabling technologies and the content. Current developments in the hardware technologies provide a renewed interest in 3-D displays both from the consumers and the display manufacturers, which is evidenced by the recent commercial products and new research results in this field.

KEYWORDS | Autostereoscopic; head-mounted displays; head tracked; multiview; parallax barrier; stereoscopic; volumetric 3-D displays

\section{INTRODUCTION}

The display industry is witnessing a major change from 2-D to realistic 3-D as evidenced by the success of recent 3-D movies and consumer 3DTV product offerings. It can be envisioned that within the next few years a major share of current displays will be replaced by 3-D displays or at least by 3 -D-capable displays and stereoscopic and autostereoscopic technologies are likely to play a major role.

The human brain extracts the depth information and perceives the stereoscopic views by fusing together the two images acquired by the eyes. The disparity in these two images (i.e., binocular disparity) is the most widely utilized visual cue in displays to render a stereoscopic view. Although this requirement was understood by Euclid, the first stereoscopic device did not appear until 1832 when Charles Wheatstone introduced the stereoscope [1]. For producing a stereoscopic image there are many technologies described in the scientific and patent literature ranging from simple anaglyph methods to advanced autostereoscopic and interactive techniques. 


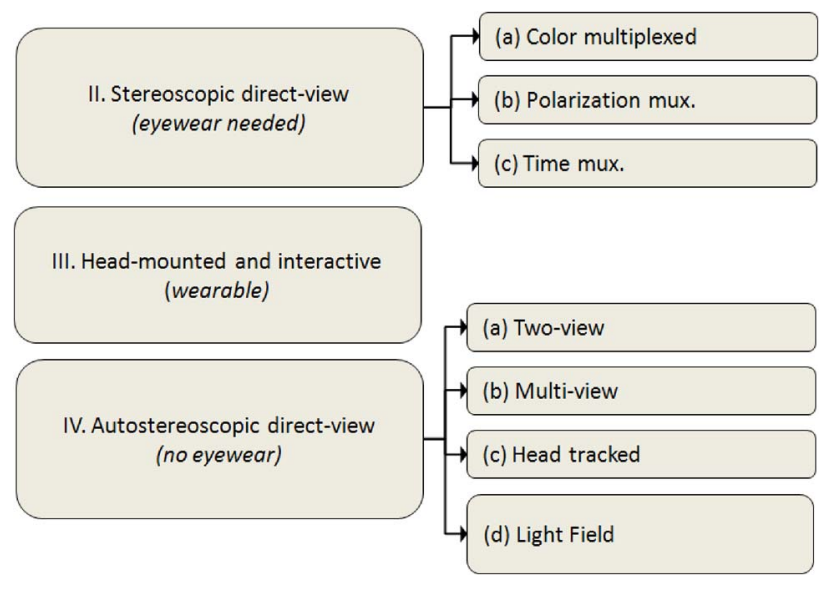

Fig. 1. Organization of the article.

Stereoscopic and autostereoscopic displays generally exhibit accommodation-convergence (AC) mismatch where the eyes converge at the apparent point of fixation in the image but focus on the screen [2], [3]. This is an unnatural condition that disrupts the correlation between vergence and focal distance. The mismatch must be kept within certain limits in order to prevent visual discomfort. As AC mismatch occurs when the virtual position of an object in the image is away from the plane of the screen there is a limit below which the mismatch is acceptable. It has been determined that the depth range should not exceed $13 \%$ of the viewing distance [4]. Other research [5] indicates that the actual tolerable conditions for comfortable viewing could be less restricted than those given in [4] and [6]. There are also several solutions suggested in the literature to overcome this limitation; these are reviewed later.

Fig. 1 provides a classification for stereoscopic and autostereoscopic technologies as well as an outline for this paper. Direct view in the section titles refers to images appearing on a flat screen, which can be a flat-panel display or a projection system. Each section provides information about how the technology works and gives an overview of the literature and state of the art and provides examples of commercially available products wherever possible. Other important categories of 3-D displays such as holographic and volumetric displays are discussed in other articles in this issue. Sections II-IV give an overview of different technologies and Section V summarizes the pros and cons of each type of 3-D display reviewed in the paper and gives author opinions about future directions.

\section{STATE OF THE ART IN DIRECT-VIEW STEREOSCOPIC DISPLAYS}

This section reviews the current status of 3-D displays, which require the viewer to wear special glasses in order to perceive 3-D.

\section{A. Color-Multiplexed Approach}

The classic example of a color-multiplexed 3-D visualization method is that using anaglyph glasses. This is an inexpensive solution to the 3-D visualization problem and can be applied whenever common color video equipment is available. Anaglyph 3-D images are produced by combining the images for the left and right eyes using a complimentary color coding technique. This method has been understood since the mid-1800s [7].

The most common anaglyph method uses the red channel for the left eye and the cyan channel for the right eye. The viewer wears a pair of colored glasses so that the left and right eyes receive the corresponding images only. The main drawbacks of this type of display are the loss of color information and the increased degree of crosstalk. The spectral response of the display and the anaglyph glasses, image compression methods and image encoding, as well as transmission protocols have been cited as sources of crosstalk [8], [129]. Anaglyph images are usually created either by capturing the images with a binocular camera or by using the depth information of the objects and only one image as recently shown [9]. For reducing the crosstalk and increasing the quality of the images, methods such as image alignment, color component blurring, and depth map adjustment have been demonstrated with significantly improved image quality [10], [11], [130]. The anaglyph viewing filters sometimes cause chromatic adaptation problems in the viewer. Commercially available NVIDIA and iZ3D drivers can output the imagery in this format [12].

ColorCode 3-D uses the color-multiplexed approach to produce full color 3-D images, which are viewed with amber and blue colored glasses. The 3-D information is written as small variations in color and the full color images have faint halos of golden and bluish tints when viewed with the naked eye [13]. The advantage is that it works with the standard display hardware and can offer full-color images at lower cost. The patented technique has also been applied for the production of movies, games, and mobile content. Ghosting and color reproduction issues have not been completely overcome in this technology.

Another display which fits in this category but offers a distinctively higher image quality is the interference filterbased method from Infitec GmbH (Ulm, Germany). Fig. 2 shows the projection scheme with these filters for producing 3-D. Two full-color projectors are fitted with narrowband interference filters, each having its own three narrow transmission bands lying within the spectral response of the red, green, and blue receptors of the eye. The right and left images are projected onto a normal diffusing screen through these filters and the eye wear with the corresponding filters separates the full-color image for left and right eyes. The crosstalk is reported to be less than $1 \%$ for the entire spectral range [14]. The technology is compatible with most of the image projection methods available but requires the application of a color gamut transformation algorithm to retain the original color 


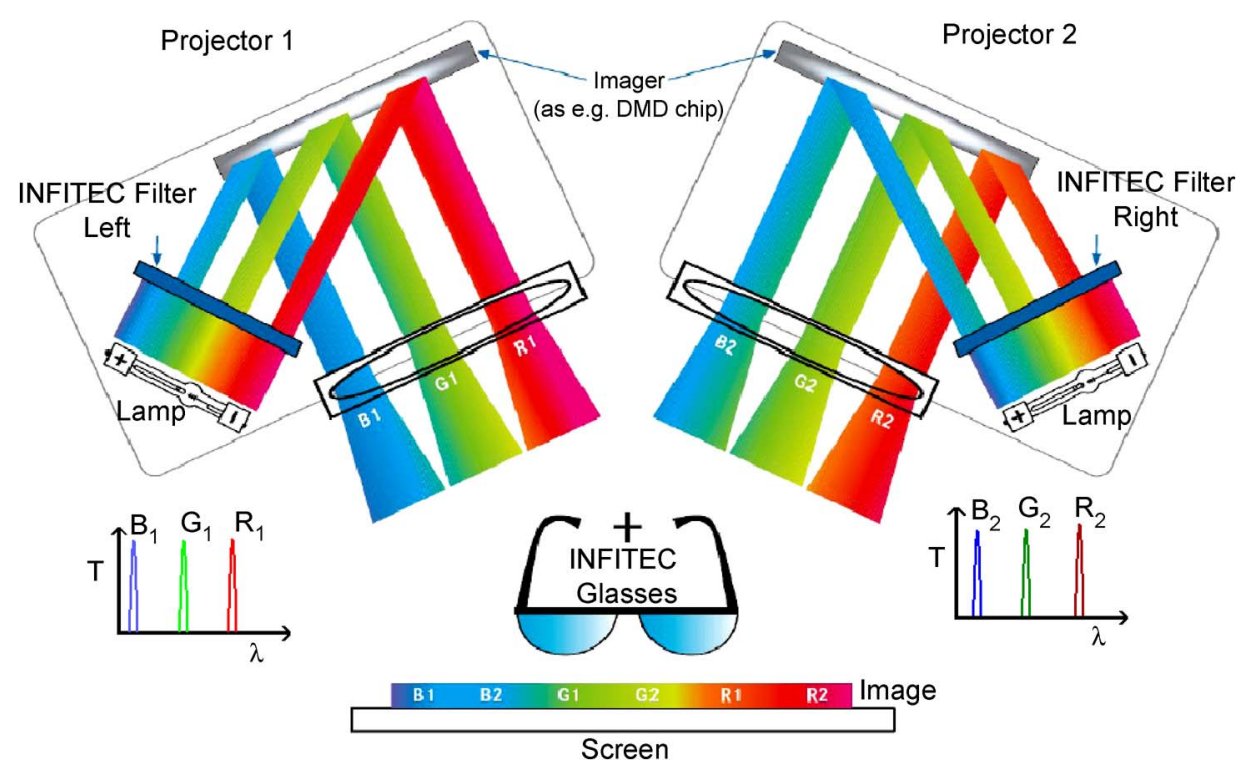

Fig. 2. Schematic setup of the Infitec system for 3-D content display. The slightly differing spectral transmission curves of the filters are also shown. From [14], with permission from The Society for Information Display.

gamut. This system has been implemented at virtual reality theaters with performance figures better than that of other passive technologies discussed above [15]. An Infitec filter wheel-based approach eliminates the cost and complexity of using two projectors and the associated alignment issues. In this case, the left and right images are presented in a time sequential fashion by a single projector and a rotating Infitec filter is used to encode the stereo pair. Images are projected onto a matte screen and the passive Infitec filters worn by the viewers separate the images.

\section{B. Polarization-Multiplexed Approach}

In this method, the state of polarization (SOP) of light corresponding to each image in the stereo pair is made mutually orthogonal. Linear or circular polarization can be employed but the latter allows more head tilt before crosstalk become noticeable. The viewer uses eyewear with appropriate polarizers to block the image not intended for that eye. Images are produced by a two-projector setup in which one projector produces either the left or right eye images with a polarization state orthogonal to that of the other projector. This method also requires the use of a special screen that preserves the SOP. For rear projection, a combination of Fresnel-lenticular surface can be used for preserving the SOP while for front projection a silver screen is used. A commercially available screen for front projection is 3-D Virtual Grey from DaLite. For rear projection, Da-Lite produces a screen called 3-D Virtual Black. Both screens are claimed to maintain $99 \%$ of polarized light and have a gain of more than 1 [16].

Another method which reduces the complexity of using two projectors uses a single projector equipped with an electrically controllable polarization rotator synchronized to the frames being displayed [17]. This method is cost effective and requires no alignment. Such a device, called ZScreen, is commercially available from RealD. This device utilizes a wire grid polarizer [18] combined with a cleanup sheet polarizer and pi cells [19] for effectively controlling the polarization direction [20]. Improved device design by providing antireflection coatings to core components, using more efficient polarizers and other modifications has been recently reported [21]. As these devices have a finite response time, blanking is necessary between the left and right images in order to reduce crosstalk; this in turn increases the light loss.

The difficulty in aligning two projectors, increased cost, and power consumption may also be eliminated by employing a single projection unit [22], [23]. In this method, light-emitting diode (LED) illumination was used for the generation of two full-color images with orthogonal polarization states by a single modulation unit containing four liquid crystal on silicon (LCOS) devices (two for each eye) and some polarization controlling optics. HDI-US Inc. (Los Gatos, CA) developed a laserbased high-definition (HD) display with a single light engine module with two high-speed LCOS devices. This rear projection display has high frame refresh rate of $360 \mathrm{~Hz}$ and a screen diagonal size of $70-120^{\prime \prime}$. It has also been claimed to outperform most of the current display technologies in terms of energy efficiency, color gamut, picture brightness, and resolution [24]. A commercial product with polarization multiplexing is the recently announced single lens 3-D projector named CF3D from LG Electronics (Seoul, Korea) [25]. 
For the flat panel display (FPD) types, available technologies are micropolarizer based ( $\mu$-pol or X-pol) [26], patterned retarder based [27], active retarder based, and dual-panel type. The first two cases work by reserving half of the total horizontal lines of pixels for the right eye and the other half for the left eye. Alternate horizontal pixel rows are orthogonally polarized by the line-interleaved micropolarizers attached to the display. Stereo pair images are displayed in a horizontally interleaved format and the passive polarizer glasses at the viewers eyes separate the images. Although it loses half of the available resolution, this system has the advantage of requiring only low-cost passive glasses.

It has also been reported that a $\mu$-pol-based display can be converted to an autostereoscopic display by adding two more $\mu$-pol sheets, where one is movable [26]. The patterned retarder approach also utilizes the same principles but uses different hardware and modified versions of the technique for higher performance by reducing polarization artifacts and dispersion [27], [28]. The optics of a patterned retarder-based display is shown in Fig. 3. Methods for fabricating these patterned retarder structures are also described in the literature [29], [30]. Display products utilizing this type of technology are available from Hyundai (Korea) and JVC (USA). The problem of limited viewing angle in the patterned retarder type displays can be resolved by reducing the glass spacing between the retarder layer and the liquid crystal layer [31], [32].

Dual-panel-type 3-D displays such as the ones from iZ3D (San Diego, CA) use two stacked liquid crystal display (LCD) panels of which one displays the images and the other controls the outgoing polarization [33]. This display is viewed with passive polarizer glasses.

Although polarizing filters can cause chromatic aberration, these types of 3-D displays offer high resolution and the color quality issues are generally negligible, unlike the anaglyph-based displays. Ghost images, hot spots, or abrupt falloff of intensity could also arise due to an improper choice of the projection lens focal length or the screen [34].

\section{Time-Multiplexed Approach}

These displays exploit the persistence of vision of the human visual system to give 3-D perception. In this technique, the left and right eye images are displayed on the screen in an alternating fashion at high frame rates, usually $120 \mathrm{~Hz}$. This is referred to as alternate frame sequencing and the viewer is required to wear battery powered active shutter glasses, which are synchronized to the content being displayed. This is done either by using an infrared emitter or by the so-called DLP Link (Texas Instruments, Dallas, TX), which uses encoded white light flashes detected by the shutter glasses in between the left and right frames. The liquid-crystal-based glasses block the left-eye images from reaching the right eye of the viewer and vice versa. An immediate disadvantage is the cost of the active shutter glasses and the excess video bandwidth required compared to 2-D. The single lens projector model PG-D45X3D from Sharp (Mahwah, NJ) utilizes the DLP Link technology for 3-D capability [35].

Time-multiplexed 3-D displays are common in the market with Samsung (Korea) and Mitsubishi (Japan) leading with their 3-D-capable rear projection TVs [36]. A time-multiplexed display with the capability of allowing the user to interact with the images has been demonstrated at Stanford University, Stanford, CA. The display, called Responsive Workbench, used head tracking to render the correct perspective [37].

Another commercially available projector with a single projection unit and time-multiplexed output is the DepthQ HD projector from Lightspeed Design, Inc. [38]. This projector uses a digital light processor (DLP) module from Texas Instruments for forming images that are viewed with active liquid-crystal-based shutter glasses. The advantages of this type of 3-D display are that they do not require a special screen and a single projection unit with a higher video bandwidth is sufficient.

The time-multiplexed approach has also been employed in immersive displays; one notable example is the Cave Automatic Virtual Environment (CAVE) system from

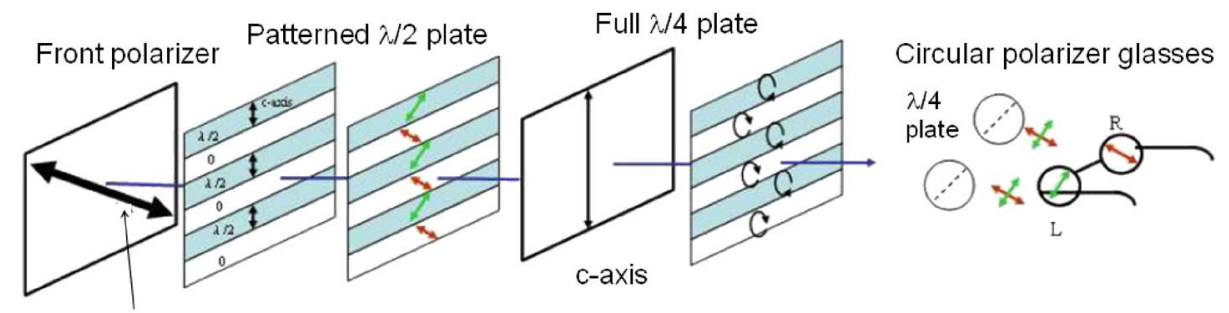

Transmission axis

Fig. 3. The structure of a patterned-retarder-type display. Linearly polarized light is used to illuminate the LCD panel with a patterned retarder plate where alternate strips offer half-wave retardation, which rotates the plane of polarization by $90^{\circ}$; the full quarter wave plate transforms the linearly polarized light into left and right circularly polarized light. At the viewer's eyes circular polarizer (combination of quarter wave plate and a linear polarizer) glasses are used to separate the views. Only half of the horizontal lines of pixels on the LCD are used to form the right or left image. From [27], with permission from The Society for Information Display. 
the University of Illinois, Chicago [39]. The system was a cube of $10 \mathrm{ft}^{3}$ in size where the walls served as screens onto which the images were rear projected. This system offered an immersive feeling to the viewer inside the cube; the development of later versions of this system has been discussed in [40].

Having considered most of the promising stereoscopic 3-D display technologies it is clear that each technology has its own advantages and limitations. For the home theater market projection systems employing a Zscreen and passive polarizer glasses could be a lower cost solution as the polarizer glasses required for each viewer are inexpensive and do not require the careful handling of the expensive shutter glasses. Possible disadvantages with this approach are the difficulty in setting up a reflective screen, the need to minimize the artifacts mentioned earlier, and ghosting effects due to the imperfect polarizers and screen.

For portable desktop displays there exists a multitude of choices employing different techniques explained before. Here also, the choice is between shutter-glassbased or polarizer-based displays. Passive glass-based dualpanel displays offer full resolution and the reduction in luminance can be compensated by using more powerful backlight sources. Frame-sequential-type displays (also known as page flipping displays) also offer full resolution over line-interleaved-type micropolarizer-based systems. Though the choice is up to the viewer, presumably one would prefer a system with low cost, high resolution, good luminance, and less sophisticated viewing aids.

\section{HEAD-MOUNTED DISPLAYS}

Developing display systems that can provide the viewer with a sense of immersion is another active topic of research. This section briefly discusses the techniques and progress in this area.

\section{A. Head-Mounted Displays (HMDs)}

Head-mounted stereoscopic displays are binocular systems with two separate image generators and they employ relay optics [41]. As these are head-worn by the user, mobility is available without interrupting the 3-D view giving a feeling of immersion in the scenes being displayed. Attempts to provide the viewer with a feeling of immersion dates back to the late 1950s when Morton Heilig implemented the Sensorama Simulator [42]. Now such systems are far more advanced and widely used for training purposes in the medical, military, and industrial fields and employ HMDs for producing the virtual environment. Augmented reality (AR) systems, a subclass of mixed reality (MR) systems, make use of see-through HMDs to enhance the viewer's understanding of the real-world information by providing him/her with computer-generated information superimposed on the real-world scenes [43]. The video see-through approach superimposes the realworld images acquired through a video camera with the

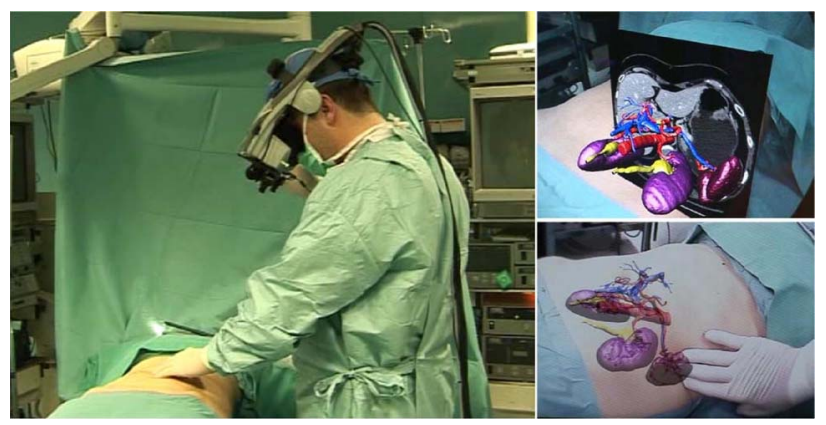

Fig. 4. An MR system setup and the mixed reality views through the HMD. From [45], with permission of IEEE.

virtual world information from a computer whereas the optical see-through method uses precision eye-pupil tracking. Thus, video see-through methods generally produce better results than the optical see-through approach where registering the real-world scene with the virtual image is difficult [44]. One of the notable advances is in the medical field where surgeons can have detailed computergenerated information superimposed on the patients in real time: the so-called computer-aided surgery (CAS) system. Ferrari et al. recently reported such an MR system (Fig. 4) in which both real-world information and computer-generated images were superimposed utilizing a video see-through approach [45].

Achieving large field of view (FOV) and high resolution simultaneously has been a problem in the case of HMDs. Optical tiling, presenting a small high-resolution inset image and a low-resolution background based on user pupil tracking, is one of the solutions proposed to address the requirement of high FOV and high resolution [46], [47]. Sensics Inc. (Columbia, MD), one of the pioneers in HMD technology, offers high-resolution displays with up to $180^{\circ}$ diagonal FOV [48]. The unit, named piSight, utilizes 12 microdisplays to create a concave wraparound display for each eye as shown in Fig. 5.

There are many HMD products currently available from different companies such as Vuzix Corporation

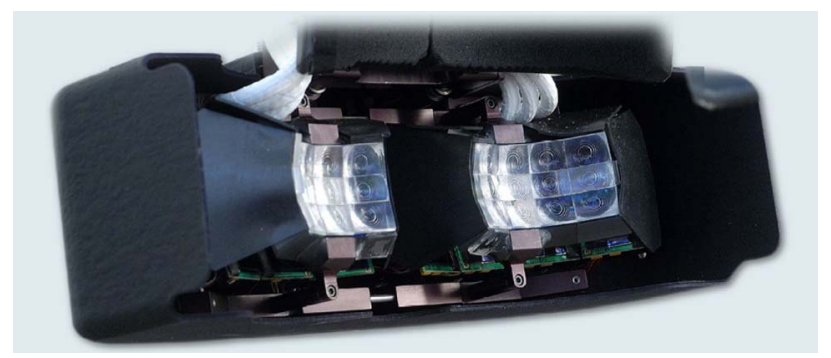

Fig. 5. The Sensics pisight HMD showing tiled microdisplays. Image courtesy of Sensics Inc. 
(Rochester, NY), Fifth Dimension Technologies (Irvine, CA), Cybermind (Maastricht, The Netherlands), i-O Display Systems LLC (Sacramento, CA), etc.

In common with other 3-D display technologies, 3-D HMDs also have the problem of AC mismatch and the problem becomes less noticeable as the image plane is moved towards infinity [5]. To solve the AC mismatch issue, systems with discrete (multifocal) or continuously variable (vari-focal) focal plane locations have been proposed [49]. The systems can be made with active optics, which forms virtual images with focal plane adjustment based on the feedback from a user-gaze detection circuit [50] or with no moving parts [51]. Optical see-through HMDs with large focal depth have been proposed for solving the AC conflict utilizing a modified Maxwellian view with increased viewing area. This method utilizes holographic optical elements and claims that 3-D images can be produced at any distance from the viewer [52]. Recently, a liquid lens with adjustable focal length has been utilized in making HMDs, which can operate either in multifocal or vari-focal mode [53]. Moving the location of the virtual image of the display screen by means of a relay lens movable along the optic axis is also a technique that was proposed to eliminate the AC mismatch problem [54].

In order to reduce the form factor of the HMD, the optical path may be folded using prism arrangements [55]. One of the main challenges associated with the development of these types of binocular 3-D display is the visual discomfort due to the differences in the left and right eye images resulting from the mismatch in the optical performance of each channel. As the human visual system depends on many visual cues and internalized patterns to interpret a 3-D scene, even the smallest errors can give rise to visual discomfort. HMDs utilize two separate displays; thus mismatch in any of the optical properties or alignment issues of the components used in the optical train of the left and right channels becomes critical [56], [57]. Refer to [58] for a discussion on the optical design problem of HMDs.

\section{STATE OF THE ART \\ IN AUTOSTEREOSCOPIC DIRECT-VIEW DISPLAYS}

Autostereoscopic displays create images with the required disparity at the exit pupils without requiring any form of special glasses or other user-mounted devices. Two-view (binocular) and multiview systems with fixed viewing zones and head/pupil tracked and super multiview (SMV) systems are different types of autostereoscopic displays discussed in detail in the following sections. In two-view displays, only a single stereo pair is displayed, whereas in multiview displays, multiple stereo pairs are produced to provide 3-D images to multiple users. In autostereoscopic displays, large Fresnel lenses, lenticular arrays, parallax barriers, or other components such as holographic elements (HOEs), mirrors, micropolarizers, and prisms are used to control light paths and to form exit pupils. In autostereoscopic displays with fixed viewing zones, these components are used to create fixed exit pupils where the user's left/right eyes must be positioned. On the other hand, head/pupil tracked displays create exit pupils according to the viewer's eye pupil locations and enable the viewer(s) to see stereo comfortably without having to remain stationary. In SMV systems, a large number of views are produced in order to create smooth motion parallax across the viewing field.

\section{A. Two-View Autostereoscopic Displays}

Two-view systems are 3-D displays where a single pair of parallax views is produced. In two-view systems, the stereo pair can be formed at a single location or the pair can repeat itself in multiple zones in space (i.e., multiviewer). The viewers' eyes have to be in the correct location within the ideal distance to perceive a stereoscopic image. Two-view systems with a head tracker can provide the same stereo pair to a single viewer or to multiple viewers and enable a greater freedom of movement.

1) Parallax Barrier Systems: LCD elements are commonly used in 3-D displays since they offer good pixel position tolerances and high position stability, have carefully controlled glass thickness, and can be successfully combined with different optical elements [59]. Image quality issues of 3-D displays based on LCD panels and solutions to improve their performance are available in the literature [60].

LCDs and other pixelated emissive displays are suitable for autostereoscopic displays as they can be combined with parallax barriers. As can be seen in Fig. 6, left and right eye image columns are placed alternately on the display and the parallax barrier, which is composed of vertical apertures separated by black masks, allows light to pass only to the desired viewing zone. The ratio of the display pixel

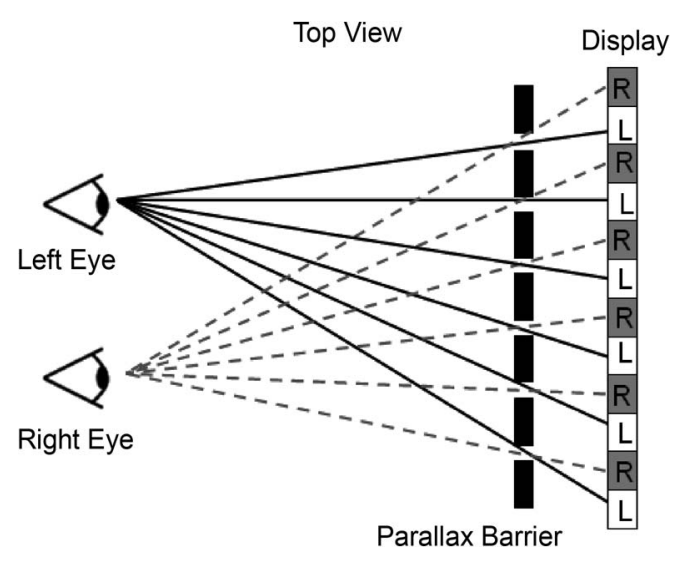

Fig. 6. Parallax barrier working principle illustration. The same stereo pair repeats itself along the viewing zone. If the parallax barrier can be turned on/off electronically, the system is 3-D/2-D switchable. 
width to the barrier pitch depends on the distance between the right and left viewing points [61]. The system described below creates repeated viewing zones along the width of the display; therefore it can also be used to create a multiview system.

The loss of brightness caused by the barriers and the loss of spatial resolution caused by using only half of the pixels for each viewing zone are the two problems associated with the parallax barrier systems. There is also a tradeoff between light efficiency and crosstalk level due to the aperture size. It has been shown that with a slanted barrier system, higher efficiency can be achieved while keeping the same crosstalk level [62]. Using aperture grills as a parallax barrier instead of black stripes on an acrylic increases the contrast by eliminating the reflection on the surface and provides better transmittance [63], [64].

In parallax barrier systems, the optimum viewing distance is proportional to the distance between the display and the parallax barrier and inversely proportional to the display pixel size. As the display resolution gets higher, the optimum viewing distance of the system gets longer. A method to improve the resolution using four views instead of two views employs parallax polarizer barriers combined with a special backlight [65].

Using two LCD elements and directing their content to two different eyes with the help of two parallax barriers is another method of increasing the spatial resolution of the parallax barrier systems. Sharp has produced a twin LCD display in which the viewing zones for left and right eyes are formed by two LCD displays where each includes a slit array and a microlens array. These are combined with a beam combiner and the images are transmitted to the viewer's corresponding eyes [66]. The display includes a head tracker and the viewing zone positions can be changed dynamically by moving the two parallax slit arrays simultaneously along the LCD plane [59].

In 1994, Sharp announced their first single-panel 3-D display [67] based on the parallax barrier method. In 1996, the design was improved with the invention of viewing position indicator (VPI). VPI is a solution to the practical problem of finding the best viewing position. As explained before, the parallax barrier system creates repeating viewing zones for left and right eyes and if the viewer's left eye is placed in a right eye viewing zone, a reversed stereoscopic (pseudoscopic) image is perceived. In systems with VPI, a few rows of pixels at the bottom of the display are used for displaying color indicators to aid the user to find the correct viewing location.

Parallax barrier systems can be 2-D/3-D switchable by removing the optical function of the parallax elements [67]. The barriers can be optically switched by using polarization-based electronic switching systems. In 2001, Sharp produced a display that could be electronically switched between two different modes [68]. In this system, the 2-D/3-D switching is achieved by the use of a patterned retarder parallax barrier, which includes a com- ponent that can rotate the polarization of light (3-D) or leave it unaffected (2-D). Crosstalk and white level variation in the 3-D mode of this display were estimated by using a model based on Fresnel diffraction [69]. The improvements in OLED technology increased their usability in 3-D displays and Samsung announced a new 14" WXGA full resolution 2-D/3-D OLED display prototype with fast switching parallax barrier [70].

Another design parameter for parallax barrier systems is the location of the parallax barrier relative to the display unit. Placing the parallax barrier at the rear of the display (between the backlight and LCD) results in lower crosstalk. On the other hand, placing the barrier in the front (between LCD and the user) results in better intensity uniformity and an optimum barrier pitch is required for reducing the crosstalk in this configuration [67].

In fixed parallax barrier systems, the pitch of the barrier is determined before the system is constructed and cannot be changed later. This causes a fixed viewing distance and viewing zone depth. When the system is used for more than one viewer with head tracking, calculation of optimum barrier parameters gets more difficult.

A solution for eliminating the fixed viewing distance and fixed viewing zone limitations can be obtained by dynamic parallax barriers instead of static ones. Dynamic parallax barrier system, Dynallax, uses a dual-stacked LCD monitor and a dynamic barrier and the system has a longer operating range. The dynamic barrier is rendered on the front display, and a modulated virtual environment, which is composed of two or four channels, is rendered on the rear display [71]. It is a 2-D/3-D switchable system, which supports two viewers with the use of a head tracker. The system has less brightness, resolution, and contrast compared to a static barrier system. Synchronization between the front and rear screens is critical for good image quality.

Parallax barriers can be used with projectors to create autostereoscopic 3-D projector systems as shown in Fig. 7 [72]. The system is composed of multiple 2-D projectors, a projection screen, and two parallax barriers. The first parallax barrier controls the output of the projectors such that the size of image pixels on the projection screen is matched with the second parallax barrier's pitch. The second parallax barrier works as a regular parallax barrier and directs the parallax images to the viewers' eyes.

2) Lenticular Systems: As can be seen in Fig. 8, lenticular systems combine cylindrical lenses with FPDs to direct the diffused light from a pixel such that it can only be seen in a certain viewing angle in front of the display. The display creates repeating viewing zones for the left and right eyes (shown with green and red colors). Similar to parallax barrier systems when the viewer's left eye is placed in a right eye viewing zone the viewer observes a pseudoscopic image. Information about efficient lenticular lens design, analysis methods, and recent advances in the technology can be found in [73] and [74]. 


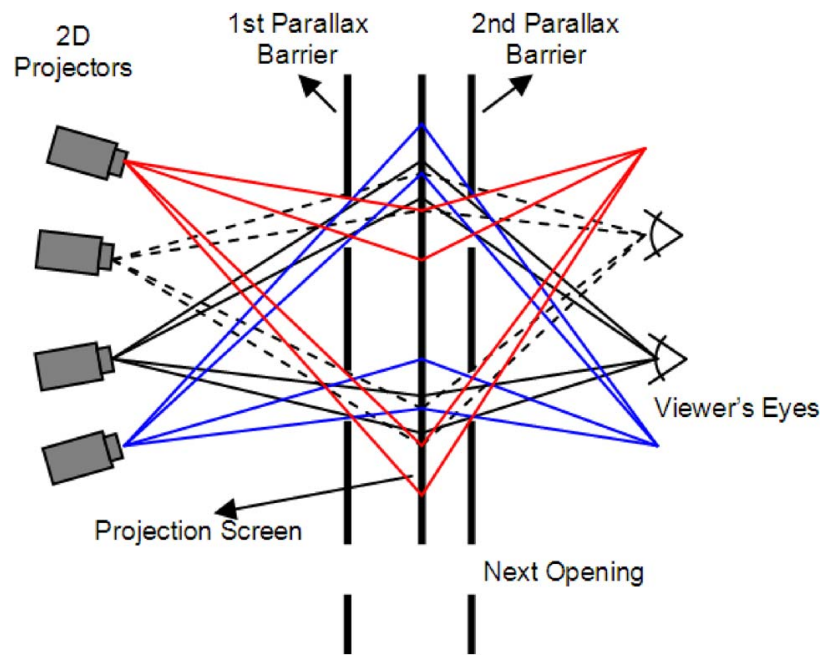

Fig. 7. Parallax-based autostereoscopic 3-D projector. The first parallax barrier controls the output of the projectors and the second parallax barrier directs the parallax images to the viewers' eyes. Adapted from [72].

The alignment of the lenticular array on the display panel is critical in lenticular systems. This alignment gets more difficult as the display resolution increases and any misalignment can cause distortions in the displayed images. Software-based solutions that compensate for the production errors can be used to improve the quality of the lenticular systems [75]. Another problem with the lenticular systems is the intensity variation along the viewing zone due to beams coming from the subpixel regions of the display panels. As the viewer changes the viewing angle, a pattern of dark and bright bands is observed. This effect can be decreased by slanting the lenses and adjusting the focal length and pitch of the lenticular array elements [76].

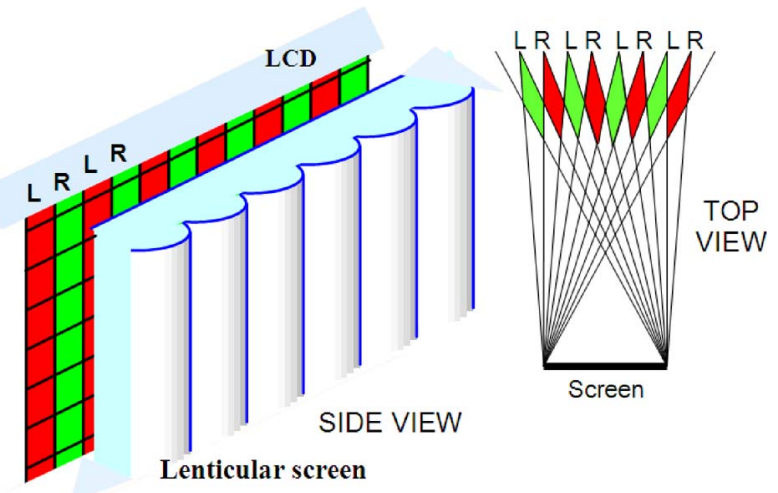

Fig. 8. Illustration of the working principle of a lenticular screen. The same stereo pair repeats itself along the viewing zone. If refraction through lens is turned on and off electronically, the system switches between 2-D and 3-D.
As explained before, 2-D/3-D switchability is a useful functionality for 3-D displays. A lenticular display can switch between two viewing modes by using lenticular lenses filled with a special material that can switch between two refracting states. Boer et al. used liquid crystal (LC)-filled lenticular lenses that can be switched electronically between refracting (3-D) and nonrefracting (2-D) modes [76]. The disadvantage of this display compared to parallax barrier switchable displays is the residual lens effects at oblique angles even when the display is working in the 2-D mode.

\section{B. Multiview Autostereoscopic Displays}

In multiview displays with fixed viewing zones, multiple different stereo pairs are presented across the viewing field. Users' left and right eyes lie in two adjacent regions that have different perspectives. The number of views in multiview displays is too small for continuous motion parallax, but various methods have been used to keep the apparent image content close to the plane of the screen and minimize the large transitions between the views [77]. Newsight GmbH (New York) produces multiview displays with reduced transition zones and suppressed glare with screen sizes up to $180^{\prime \prime}$ (the world's largest) [78].

As discussed before, static parallax barriers combined with pixelated emissive displays create repeated viewing zones. In multiview parallax barrier systems, more than two images can be created in the viewing zone to produce motion parallax. Sanyo has produced a multiview image integration system such that seven parallax video images can be combined together into a single video that can fit into the special step barrier they developed [79], [80].

Endo et al. used parallax barriers to create a cylindrical 3-D display that is based on parallax panoramagram to show 3-D images to multiple viewers from $360^{\circ}$ of arc horizontally without 3-D glasses [81]. A cylindrical parallax barrier and a rotating 1-D light source array are used in an experimental cylindrical display system with the resolution of 1254 pixels circularly and 128 pixels vertically. Each pixel has a $60^{\circ}$ viewing angle that is divided into 70 views to create a smooth parallax around the display.

Time multiplexing is another method that can be used to produce multiview autostereoscopic displays with parallax barriers. Choi et al. fabricated a 3-D display with increased viewing zones by using dynamic barriers located between the lens array and the display panel [82].

Lenticular arrays combined with pixelated emissive displays can also be used to create multiview 3-D displays. The problem with lenticular systems is the reduction in resolution with the increase in the number of viewers. In vertically aligned lenticular arrays, the resolution decreases in the horizontal direction only, however if the lenses are slanted the resolution loss is distributed into two axes [76]. Philips (Eindhoven, The Netherlands) produced a slanted lenticular screen that can support 7-15 views 


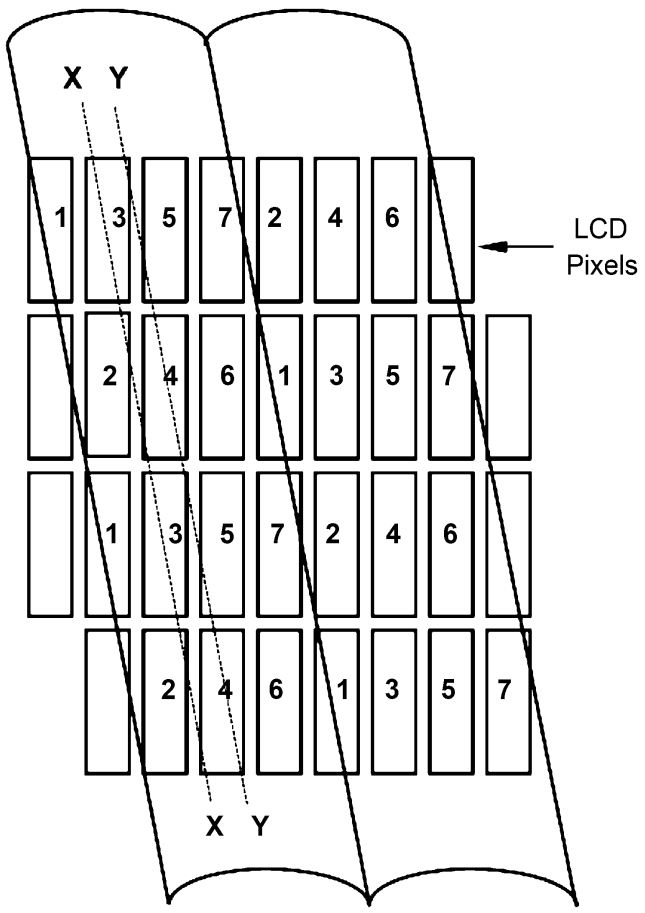

Fig. 9. Illustration of the working principle of a slanted lenticular screen. LCD is located one focal length behind the lenticular sheet. From [83], with permission from SPIE.

depending on model, and the structure of the screen is shown in Fig. 9 [83].

The figure shows the relationship between the pixels and the slanted lenticular sheet for a seven-view display. As the LCD is located in the focal plane of the lenticular sheet, the horizontal position on the LCD corresponds to the viewing angle. Therefore, all points on line XX direct view 3 in a given direction, and all points on line YY direct view 4 in another direction. The way in which the effect of flipping is reduced is evident by examining line $\mathrm{XX}$ where view 3 predominates, but with some contribution from views 2 and 4. Similarly, for the angle corresponding to line YY, view 4 with some contribution from views 3 and 5 is seen.

Implementing autostereoscopic methods in mobile displays is a promising field of study due to increased public interest in 3-D displays. Sharp introduced the first mobile phone with 3-D capability in early 2000 and sold more than 1 million units [84]. Different mobile 3-D approaches can be found in the literature. Bilkent University, New York, NY, leads a consortium of academic and industrial groups that address various aspects of 3-D mobile phone technology [85]. In a recent study, an autostereoscopic 3-D solution for mobile displays has been demonstrated by using 3-D film, directional backlight, and an LCD panel. In this time-sequential autostereoscopic 3-D display approach, the two different views created by changing the direction of the light in a light guide are directed to two different eyes by using a specially designed
3-D film [86]. New directional backlights for autostereoscopic display applications that can be scanned $16^{\circ}$ have also been announced [87]. The multiview approach can also be applied to mobile displays. The images captured by a calibrated multi-imager camera array can be projected onto a retroreflective screen by using multiprojectors [88]. In this configuration, multiple views can be created along the viewing field. Special screens play an important role in mobile 3-D displays [89].

\section{Head-Tracked Displays}

Head-tracked displays can be divided into the following principal categories: Fresnel lens, lenticular, projection, and parallax barrier. In addition, other techniques including prismatic and holographic can be employed.

In the 1990s, Sharp Laboratories of Europe developed a system where the images from two LCDs are combined with a semisilvered mirror [90]. A display operating on a similar principle was described by the Japanese Sea Phone company [91] where the illumination is provided from two monochrome displays, and the Massachusetts Institute of Technology (MIT) Media Lab described the use of an SLM that rotates the polarization by $90^{\circ}$ in order to produce exit pupils [92]. The Korea Institute of Science and Technology display projects the images of two LCD panels on to a Fresnel lens [93].

The simplest lenticular screen method is to place a screen with vertical lenses in front of an LCD and swap the images on the pixel rows to ensure that the eyes never see pseudoscopic images [94]. Sharp developed a display where a novel LCD mask structure provides contiguous light across the viewing field [59]. The majority of projection stereoscopic displays operate on the principle of forming real images of the projector lenses in the viewing field.

The four main classes of projection display are determined by the screen type. These are all described in [95] and are: retroreflecting [96], [97], double lenticular screen [98], [99], mirror [100], and lens [101].

Parallax can be used in various ways in a head-tracking display. In a method proposed by Hattori [102], a parallax barrier was used to block the light from a pair of projectors that form exit pupils by utilizing a large convex mirror. In the Varrier display of the University of Illinois, a physical parallax barrier with vertical apertures is located in front of the screen [103]. Light is steered with the use of a dynamic virtual barrier. The parallax display of New York University (NYU), New York, uses a fast dynamic parallax barrier located in front of the display [104].

There are several methods of producing a head-tracking display; among these are prismatic arrays, macrolens arrays, and HOEs. Dresden University, Dresden, Germany, has developed a display that replaces a parallax barrier with a prism array [105]. Exit pupils can also be produced by HOEs [106].

The single-user Free2C display developed at Fraunhofer Heinrich Hertz Institute (HHI) overcomes the problems of 
restricted viewer movement by using head tracking to make the viewing sweet spot follow the position of the head [107]. A head tracker determines this position and controls a vertically aligned lenticular screen located in front of an LCD to enable the viewer movement in both $\mathrm{X}$ and $\mathrm{Z}$ directions. It achieves this by moving the lenticular screen, also in the $\mathrm{X}$ and $\mathrm{Z}$ directions, with mechanical actuators. This display can also be incorporated into a kiosk (Fig. 10) where user's gestures are recognized; this enables simple intuitive handling of objects within the image. Threedimensional objects floating in front of the display can be rotated by using gestures, and virtual buttons can be pressed simply by pointing at them (virtual 3-D touch screen). The Free2C kiosk is perfectly suited for applications in high-tech showrooms, convention halls, airports, shopping malls, and department stores.

The SeeFront company in Germany produces a singleuser $22^{\prime \prime}$ head-tracked display that, like the Fraunhofer HHI display, uses a lenticular screen in front of an LCD [108]. In this case, there are no moving parts and the images are directed to the viewer's eyes by switching the LCD subpixels.

There are also holographic and hybrid display solutions with viewer tracking where both conventional and holographic technologies are employed such as the SeeReal display system [109]. The MUTED display system [110] developed within a European-Union-funded project is also a hybrid approach and uses a multiuser head tracker, and its output is a spot pattern that is converted by the optical array into a series of collimated beams that intersect at the viewers' eyes. The intersection regions are referred to as exit pupils and are capable of movement over a large region.

A multiviewer laser-based 3-D autostereoscopic display is under development in the HELIUM3D project [111], [112]. Fig. 11 is a simplified schematic diagram. The system

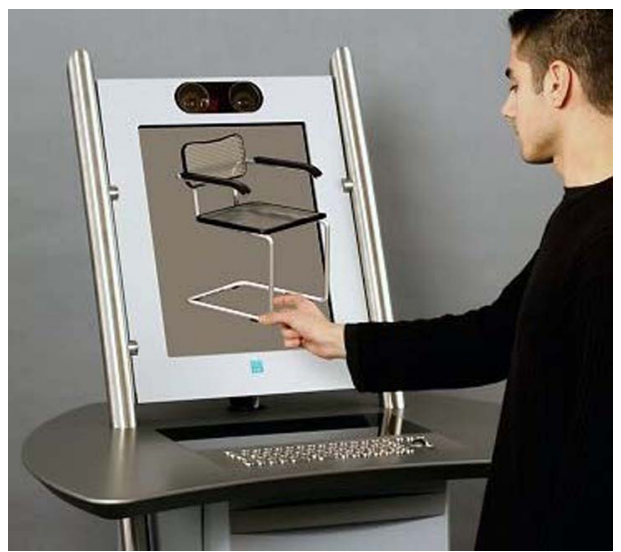

Fig. 10. Free2C interactive kiosk. User handling a multimedia application on the computer without using keyboard or mouse made possible with contact-free interaction technology and autostereoscopic displays.

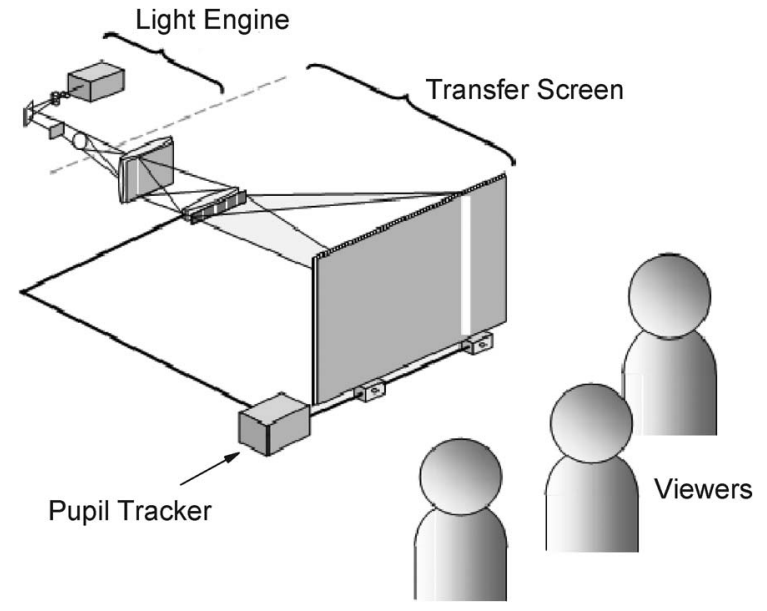

Fig. 11. HELIUM3D simplified schematic diagram of display. Multiple exit pupils are formed dynamically by controlling light directions with an SLM during the period of a horizontal scan [111], [113]. The pupil tracker uses digital cameras and custom software to acquire and track user's eye pupil locations and serves as the dynamic feedback for adjusting the exit pupil locations. Reprinted with kind permission of the HELIUMBD consortium, which the authors are part of.

does not incorporate a flat panel display and image information is supplied by a fast light valve (LV) that controls the output of red, green, and blue (RGB) lasers. The high display frame rate permits many modes of autostereoscopic operation to multiple mobile viewers due to the ability of the display to present a different image to every eye.

\section{Light Field Displays}

1) Super Multiview: An SMV display is a multiview display where the number of discrete images presented is sufficiently large to give the appearance of continuous motion parallax [114]. There is no AC mismatch and research has been carried out into the number of views required for this condition. According to Takaki from Tokyo University of Agriculture and Technology, Tokyo, Japan, when the angle pitch of the directional rays is $0.2^{\circ}-0.4^{\circ}$, more than two directional rays passing through a point in 3-D space enter the viewer's eye simultaneously so that the viewer's eye can focus on that point as illustrated in Fig. 12 [115]. No discrepancy appears between the convergence function and the accommodation function so that the display does not induce visual fatigue. Takaki presents an SMV projection configuration with 64 views on a 9.2-in screen size.

A system has been demonstrated at CeBIT 2010 by Sunny Ocean Studios (Singapore) [116] that can supply 64 viewing zones. This capability qualifies this as being SMV but still does not quite fulfil the criteria set out in the references for smooth motion parallax.

In some other types of displays, discrete beams of light that vary with angle radiate from each point on the screen. 


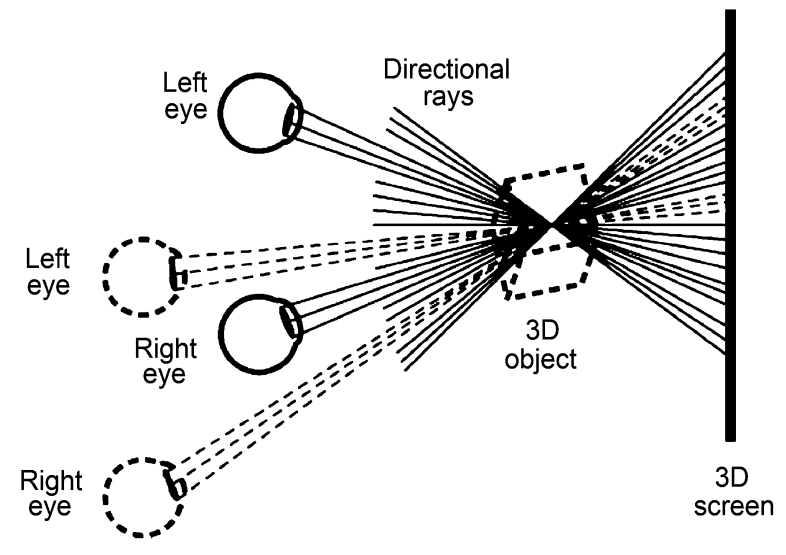

Fig. 12. Three-dimensional supermultiview images displayed with high-density directional rays (reproduced from [115] with permission from IEEE).

These can take two forms. In the first type, optical modules provide multiple beams that either converge and intersect in front of the screen to form real image "voxels" or diverge to produce virtual "voxels" behind the screen (Fig. 13). The screen diffuses the beams in the vertical direction only allowing viewers vertical freedom of movement without altering horizontal beam directions. As the projectors/ optical modules are set back from the screen, mirrors are situated on either side in order to provide virtual array elements on either side of the actual array.

Each screen pixel has a number of discrete emission angles controlled by the image generators behind the screen. Although this class of display is not volumetric, the

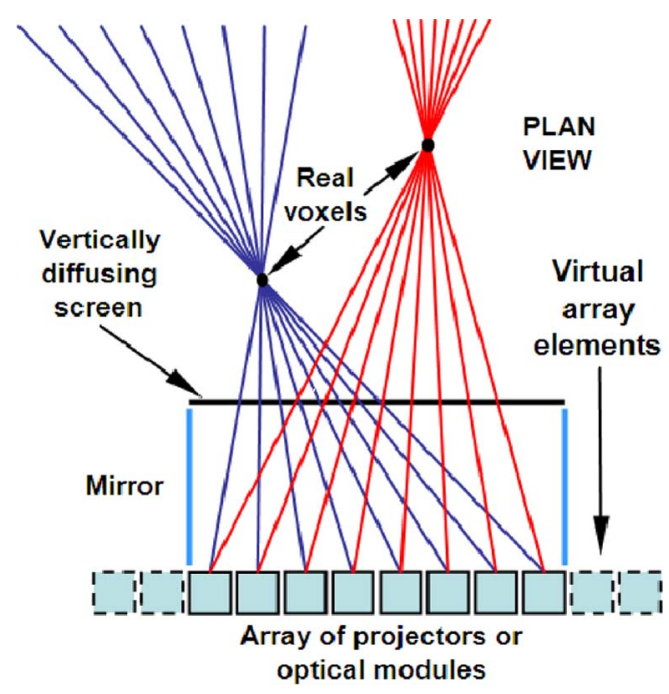

Fig. 13. Holographika display. Multiple beams either converge and intersect in front of the screen to form real image "voxels" or diverge to produce virtual "voxels" behind the screen.

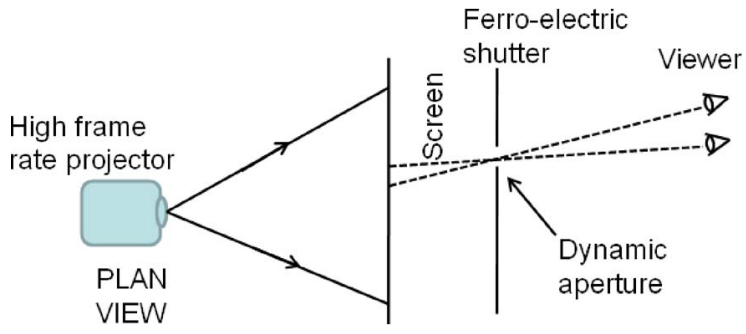

Fig. 14. Dynamic aperture display. A horizontally scanned vertical dynamic aperture directs the light from images produced on a fast projector located behind it.

term "voxel" is used to refer to the regions of image space over which an apparent solid pixel is formed.

Examples of this type of display are Holografika (Budapest, Hungary) [117], QinetiQ (Hampshire, U.K.) [118], and Koç University, Istanbul, Turkey [119]. Holografika refers to its display as pseudoholographic display and can currently supply a $32^{\prime \prime}$ display using 9.8 megapixels and a 72" version with 34.5 megapixels [120]. The QinetiQ display (see Fig. 14) appears to operate on the same principles but uses projectors instead of the optical modules of Holografika. Koç University also developed a concept based on using an array of scanners made of standard printed circuit board (PCB) material [121], where each module carries an array of LEDs on the moving platform to replace the array of microdisplay panels used by Holografika (illustrated in Fig. 15) [119]. Another type of display uses a fast frame-rate projector in conjunction with a horizontally scanned dynamic aperture as in Fig. 14. Although the actual embodiment appears to be totally different from the multiple beam approach, the effect is the same; in the case of the dynamic aperture the beams are

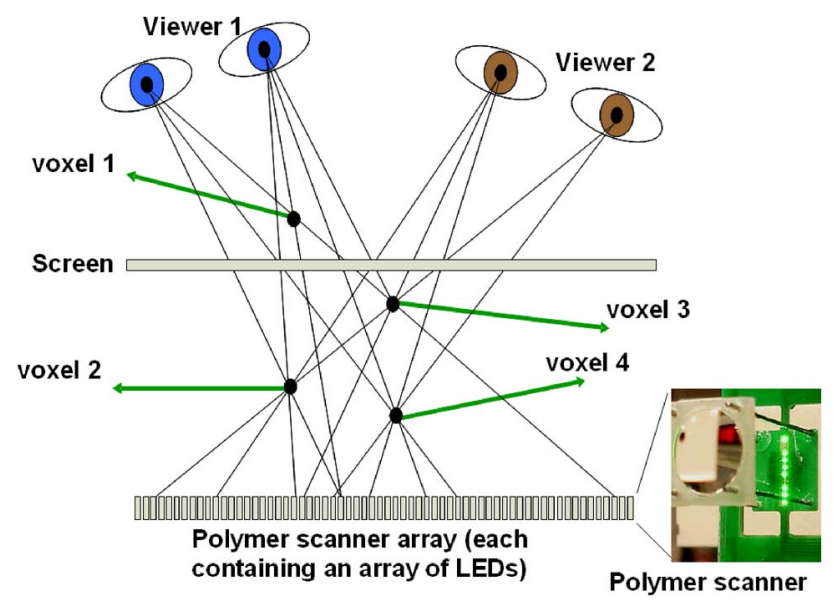

Fig. 15. Display developed at Koç University using a large array of FR4 scanners [121] integrated with LED array and lenses. 
formed in temporal manner. An early version of this type of display used a mechanically scanned aperture with the images supplied by a cathode ray tube [122]. More recently, this has been developed further at Cambridge University, Cambridge, U.K., with the use of a fast digital micromirror device (DMD) projector and a ferroelectric shutter [123]. Kajiki developed a concept called focused light array that uses an array of light sources and scanners to create an SMV display [124].

Integral imaging displays, an early light field technique first proposed in 1908, use an array of small lenses used to produce a series of elemental images in their focal planes. If the lens array is a 2-D fly's eye lens, then motion parallax in both horizontal and vertical directions is provided.

NHK, Japan, has carried out research into integral imaging for several years; this includes one approach using projection [125]. In 2009, NHK announced it had developed an "integral 3DTV" achieved by using a 1.34-mm pitch lens array that covers an ultrahigh definition panel. Hitachi has demonstrated a 10" "full parallax 3-D display" that has a resolution of $640 \times 480$. This uses 16 projectors in conjunction with a lens array sheet [126] and provides vertical and horizontal parallax. There is a tradeoff between the number of "viewpoints" and the resolution; Hitachi uses sixteen $800 \times 600$ resolution projectors and in total there are 7.7 million pixels (equivalent to a resolution of $4000 \times 2000$ pixels).

\section{CONCLUDING REMARKS AND THE FUTURE OF 3-D DISPLAYS}

Three-dimensional displays have many applications and a large range of 3-D demonstrators and products have recently been introduced to the market. This section provides the authors' opinions about the future research directions with the conviction that 3-D display users are unlikely to accept a compromise on image quality and resolution while switching from 2-D to 3-D displays.

One of the important requirements for wide acceptance from the users is that products must allow seamless switching between 3-D and 2-D modes. Though choice is up to the viewer, presumably one would prefer a system with no restriction on user movement, low cost, high resolution, good brightness, and absence of (or at least low cost and maintenance-free) viewing aids. Visual discomfort due to asymmetries in brightness, geometry, and imperfect temporal parallax are also issues that need to be addressed. Crosstalk is another serious artifact present in almost all types of stereoscopic displays but this can be brought to an acceptable level by employing suitable image processing algorithms and optimized display and capture hardware. A display technique with zero crosstalk and acceptable image quality is still not available.

1) Stereoscopic Direct-View Technologies: Anaglyph methods are simpler than others; however, the image quality is not sufficient for wide adoption due to the poor color reproduction and crosstalk issues. These technologies are likely to be limited for distribution of 3-D pictures in printed media and as multimedia content that can be seen on regular 2-D TV sets with simple and low-cost glasses. Polarization-based 3-D displays offer important advantages for the eyewear as they are very inexpensive, lightweight, and passive (no batteries).

In the stereoscopic category, high resolution and brightness can be obtained from both polarizationmultiplexed displays and the time-multiplexed shutter glasses-based displays. As an example, dual-panel architecture (e.g., iZ3D), or those that use dual projectors can have full resolution without any compromise in brightness. In terms of the eyewear, passive glasses have the advantage as they are simple and lightweight, do not require battery replacement, and are low cost.

Polarization-based flat panel displays ( $\mu$-pol, active retarder, etc.) typically lose half of the spatial resolution to produce a stereo pair image. Furthermore, when those displays are used in 2-D mode, 50\% of the light is lost to polarization components. When patterned micropolarizers can be integrated into the panel production, the system can be made more light efficient. Users are not likely to give away the high-resolution and brightness advantages to go from 2-D to 3-D. Therefore, shutter-glass-based systems have a resolution advantage over the polarization-based systems. The drawbacks of the shutter-glass-based systems are the requirement for faster LCD/LCOS switching times and more complex and heavier shutter glasses that need battery replacement. In this case, the major cost of the 3-D capability is placed in the high-speed panels, electronics, and eyewear. Which approach is better for an application depends on many factors; passive eyewear may be more favorable for applications where many people or long duration use is involved.

For 3-D projection displays (rear projection and front projection), full HD resolution can be maintained by utilizing two projectors or a time-multiplexing scheme with the help of a single-pixel polarization rotator in front of the projection lens. The approach from HDI, for example, can be considered a hybrid between temporal and polarization multiplexing. It features a dual-panel projection engine that allows polarization glass-based rear projection system and employs high-speed LCOS devices and RGB laser illumination. The system provides 2-D/3-D capability and uses simple low-cost polarization glasses without sacrificing resolution or brightness.

2) Head-Mounted Systems: HMD optical design is challenging due to the wide FOV and large exit pupil size requirements in high-resolution displays. The size and weight of HMDs are often quite large and the user comfort is typically low. Displaying 3-D images on HMDs brings additional challenges with subpixel accuracy requirements in registration of images and optical distortion. Gaming is 
one important area for 3-D HMDs and there are already successful products with a large FOV.

3) Autostereoscopic Technologies: For two-view and multiview systems, simple 2-D/3-D switching should be possible for a winning technology. For mobile and computer monitor applications (e.g., gaming, CAD/CAM design, kiosk browsing, etc.), two-image systems should be sufficient and multiview capability is not needed. A stereo image pair that can change dynamically with the headtracked viewer head position could also be a winning combination. For TV, cinema, or table-top 3-D displays, multiview and multiviewer capabilities are important, but current systems do not meet the needs of these segments.

4) Autostereoscopic Head-Tracked Displays: These enable tracking of the viewing regions to follow the viewers' eyes so that a pair of images is sufficient to display 3-D with both horizontal and vertical parallax to the viewer. Motion parallax can be achieved by presenting only as many images as there are viewer eyes. This approach may require dynamic optics/optomechanics, but it avoids displaying large amounts of redundant information in contrast to holographic displays. However, the processing required to render images in accordance with the viewers' eye positions is challenging. At the present time, head-tracked displays appear to provide a solution for multiviewer 3-D until a fully holographic display is available.

5) Autostereoscopic Supermultiview Systems: These displays provide multiview and multiviewer capabilities but they require more complex hardware and software compared to two-view systems. SMV displays have the potential to solve $\mathrm{AC}$ mismatch in order to provide more realistic true 3-D display system. Providing two or more views within each eye pupil can be done more easily if the pupil is tracked and the light beams are redirected using dynamic optics in the display system. If tracking is not employed, a large amount of information must be displayed in order to provide true continuous motion parallax with no AC mismatch. Currently, display panels do not have sufficient native resolution to give this amount of information without having to resort to the use of complex display configurations. More research and development is needed in this area. Early adaption of those technologies can be in advertising, simulators, and gaming kiosks where higher costs could be afforded.

6) Screen Technologies: Pico and mobile projectors are advancing rapidly and there are already examples of 3-D mobile displays and this seems to be an important future direction. We believe screen technologies that can form separate viewing zones for each pupil are essential in order to expand the capabilities of tiny projectors to 3-D. Note that laser-based pico projectors are currently limited to about ten lumens. Gain and ambient rejection capabilities of screens will be critical for all battery operated devices. Good candidate screen technologies for 3-D mobile displays can utilize microlens arrays and retroreflectors [127], [128]. We expect to see a lot of research activity in the area of screens with special features.

\section{Acknowledgment}

The authors would like to thank the HELIUM3D consortium members. Feedback and comments from C. Chinnock and K. Werner from Insight Media are gratefully acknowledged.

\section{REFERENCES}

[1] I. Sexton and P. Surman, "Stereoscopic and autostereoscopic display systems," IEEE Signal Process Mag., vol. 16, no. 3, pp. 85-99, May 1999.

[2] P. Lelyveld, Basic Visual Perception Concepts Related to 3D Content, pp. 1-8, Accessed Sep. 8, 2010. [Online]. Available: http:// www.etcenter.org/files/public/ 090617_3D_primer.pdf

[3] G. D. Love, D. M. Hoffman, P. J. W. Hands, J. Gao, A. K. Kirby, and M. S. Banks, "High-speed switchable lens enables the development of a volumetric stereoscopic display," Opt. Exp., vol. 17, pp. $15716-15725$ 2009.

[4] H. Choi, J. Kim, J. B. Park, and B. Lee, "Analysis on the optimized depth of 3D Displays without an accommodation error," in Dig. Int. Meeting Inf. Display, 2007, pp. 1811-1814.

[5] D. M. Hoffman, A. R. Girshick, K. Akeley, and M. S. Banks, "Vergence-accommodation conflicts hinder visual performance and cause visual fatigue," J. Vis., vol. 8, pp. 1-30, 2008.
[6] W. A. Ijsselsteijn, P. J. H. Seuntiëns, and L. M. J. Meesters, Human Factors of 3D Displays, O. Schreer, P. Kauff, and T. Sikora, Eds. New York: Wiley, 2005.

[7] W. Rollman, "Zwei neue stereoskopische methoden," Annalen deer Physik und Chemie, vol. 90, pp. 186-187, 1853.

[8] A. J. Woods and T. Rourke, "Ghosting in anaglyphic stereoscopic images," in Proc. SPIE-Stereoscopic Displays and Virtual Reality Systems XI, A. J. Woods, J. O. Merritt, S. A. Benton, and M. T. Bolas, Eds., 2004, pp. 354-365.

[9] F. Matsuura and N. Fujisawa, "Anaglyph stereo visualization by the use of a single image and depth information," J. Vis., vol. 11, pp. 79-86, 2008.

[10] I. Ideses and L. Yaroslavsky, "New methods to produce high quality color anaglyphs for 3-D visualization," Proc. Image Anal. Recog., vol. 3212, A. Campilho and M. Kamel, Eds. Berlin, Germany: Springer-Verlag, 2004, pt. 2, pp. 273-280.

[11] I. Ideses and L. Yaroslavsky, "Three methods that improve the visual quality of colour anaglyphs," J. Opt. A, Pure Appl. Opt., vol. 7, pp. 755-762, 2005.
[12] iZ3D, Accessed 19 Sep. 2010. [Online]. http://www.iz3d.com/

[13] S. E. B. Sorensen, P. S. Hansen, and N. L. Sorensen, "Method for recording and viewing stereoscopic images in color using multichrome filters," U.S. Patent 6687 003, 2004.

[14] H. Jorke, A. Simon, and M. Fritz, "Advanced stereo projection using interference filters," J. Soc. Inf. Display, vol. 17, no. 5, pp. 407-410, 2009.

[15] D. Gadia, C. Bonanomi, M. Rossi, A. Rizzi, and D. Marini, "Color management and color perception issues in a virtual reality theater," in Proc. SPIE, A. J. Woods, N. S. Holliman, and J. O. Merritt, Eds., 2008, no. 1, p. 68030S.

[16] DaLite, Accessed Feb. 25, 2010. [Online]. Available: http://www.da-lite.com/

[17] D. W. G. Byatt, "Stereoscopic television system," U.S. Patent 4281 341, 1981.

[18] J. J. Wang, F. Walters, X. Liu, P. Sciortino, and X. Deng, "High-performance, large area, deep ultraviolet to infrared polarizers based on $40 \mathrm{~nm}$ line/78 $\mathrm{nm}$ space nanowire grids," Appl. Phys. Lett., vol. 90, no. 6, pp. 061104-061106, 2007. 
[19] P. J. Bos and K. R. Koehler, "The pi-cell: A fast liquid-crystal optical-switching device," Molecular Crystals Liquid Crystals, vol. 113, pp. 329-339, 1984.

[20] L. Lipton and M. Cowan, "Zscreen modulator with wire grid polarizer for stereoscopic projection," U.S. Patent $7633666,2009$.

[21] M. Cowan, J. Greer, L. Lipton, and J. Chiu, "Enhanced Zscreen modulator techniques," U.S. Patent 7477 206, 2009.

[22] L. Bogaert, Y. Meuret, B. Van Giel, H. Murat, H. De Smet, and H. Thienpont, "Projection display for the generation of two orthogonal polarized images using liquid crystal on silicon panels and light emitting diodes," Appl. Opt., vol. 47, no. 10, pp. 1535-1542, 2008.

[23] L. Bogaert, Y. Meuret, B. V. Giel, H. D. Smet, and H. Thienport, "Design of a compact projection display for the visualization of 3-d images using polarization sensitive eyeglasses," J. Soc. Inf. Display, vol. 17, no. 7, pp. 603-609, 2009.

[24] HDI US Inc., Accessed Feb. 25, 2010. [Online]. Available: http://www.hdi3d.com/ technology.html

[25] LG Electronics, LG Electronics Thinks Outside of the Box: Unveils World's First Full HD 3D Projector, Press Release, Jun. 1, 2010.

[26] S. M. Faris, "Novel 3D stereoscopic imaging technology," Proc. SPIE-Int. Soc. Opt. Eng., vol. 2177, no. 1, pp. 180-195, 1994.

[27] Y.-J. Wu, Y.-S. Jeng, P.-C. Yeh, C.-J. Hu, and W.-M. Huang, "20.2: Stereoscopic 3D display using patterned retarder," in Dig. Tech. Papers SID Symp., 2008, vol. 39, no. 1, pp. $260-263$.

[28] P.-C. Yeh, C.-W. Chen, C.-I. Huang, Y.-J. Wu, C.-H. Shih, and W.-M. Huang, "P-83: An improvement of color difference between two eyes of stereoscopic display with glasses," in Dig. Tech. Papers SID Symp., 2009, vol. 40, no. 1, pp. 1431-1433.

[29] M. E. Sousa, G. P. Crawford, C. W. M. Bastiaansen, and D. J. Broer, "P-165: Use of lyotropic liquid crystals for patterned polarizer and retarder applications," in Dig. Tech. Papers SID Symp., 2006, vol. 37, no. 1, pp. 836-839.

[30] L. Lin, Y.-C. Chen, C.-H. Tsai, and K. Lee, "A method of fabricating micro-retarder plates by a laser system," Proc. SPIE-Int. Soc. Opt. Eng., vol. 6803, no. 1, 2008, $68031 \mathrm{H}$.

[31] G.-F. Tseng, C.-H. Tsai, H. Y. Lin, W.-J. Huang, K.-C. Huang, and K. Lee, "The fabrication of microretarder for in-cell stereoscopic LCD using reactive liquid crystal," Proc. SPIE-Int. Soc. Opt. Eng., vol. 6490, no. 1, 2007, 64900W.

[32] S. J. Lee, M. J. Kim, K. H. Lee, and K. H. Park, "Review of wire grid polarizer and retarder for stereoscopic display," Proc. SPIE-Int. Soc. Opt. Eng., vol. 7237, no. 1, 2009, 72370P.

[33] A. Lukyanitsa, "Composite dual LCD panel display suitable for three dimensional imaging," U.S. Patent 7342 721, 2008.

[34] B. Brubaker, 3D and 3D Screen Technology, 3D@Home Consortium Publication, 2009.

[35] Sharp, PG-D45X3D, Accessed Mar. 30, 2010. [Online]. Available: http://www. sharpusa.com/
[36] C. Chinnock, 3D Coming Home in 2010, 3D@Home Consortium Publication, 2009.

[37] W. Krüger, C.-A. Bohn, B. Fröhlich, H. Schüth, W. Strauss, and G. Wesche, "The responsive workbench: A virtual work environment," Computer, vol. 28, no. 7, pp. 42-48, 1995.

[38] LightSpeed, Accessed Mar. 1, 2010. [Online]. http://www.depthq.com/

[39] C. Cruz-Neira, D. J. Sandin, T. A. DeFanti, R. V. Kenyon, and J. C. Hart, "The CAVE: Audio visual experience automatic virtual environment, "Commun. ACM, vol. 35 , no. 6 , pp. 64-72, 1992.

[40] J. Leigh, A. Johnson, L. Renambot, T. DeFanti, M. Brown, B. Jeong, R. Jagodic, C. Krumbholz, D. Svistula, H. Hur, R. Kooima, T. Peterka, J. Ge, and C. Falk, "Emerging from the CAVE: Collaboration in ultra high resolution environments," in Proc. 1st Int. Symp. Universal Commun., 2007, pp. 96-99.

[41] S. Pastoor and M. Wöpking, "3-D displays: A review of current technologies," Displays, vol. 17, pp. 100-110, 1997.

[42] M. Heilig, "Sensorama simulator," U.S. Patent, Aug. 28, 1962.

[43] R. Azuma, Y. Baillot, R. Behringer, S. Feiner, S. Julier, and B. MacIntyre, "Recent advances in augmented reality," IEEE Comput. Graph. Appl., vol. 21, no. 6, pp. 34-47, Nov./Dec. 2001.

[44] E. Edwards, J. Rolland, and K. Keller, "Video see-through design for merging of real and virtual environments," Proc. IEEE Annu. Int. Symp. Virtual Reality, Sep. 1993, pp. 223-233.

[45] V. Ferrari, G. Megali, E. Troia, A. Pietrabissa, and F. Mosca, "A 3-D mixed-reality system for stereoscopic visualization of medical dataset," IEEE Trans. Biomed. Eng., vol. 56, no. 11, pp. 2627-2633, Nov. 2009.

[46] E. M. Howlett, "High-resolution inserts in wide-angle head-mounted stereoscopic displays," Proc. SPIE-Int. Soc. Opt. Eng., vol. 1669, no. 1, pp. 193-203, 1992.

[47] J. P. Rolland, A. Yoshida, L. D. Davis, and J. H. Reif, "High-resolution inset head-mounted display," Appl. Opt., vol. 37, no. 19, pp. 4183-4193, 1998.

[48] Sensics, "Sensics-Lightweight panoramic head-mounted displays," Accessed Sep. 19, 2010. [Online]. Available: http:// sensics.com/

[49] S. Liu and H. Hua, "A systematic method for designing depth-fused multi-focal plane three-dimensional displays," Opt. Exp., vol. 18, no. 11, pp. 11 562-11 573, 2010.

[50] T. Sugihara and T. Miyasato, "32.4: A lightweight 3-D HMD with accommodative compensation," in Dig. Tech. Papers SID Symp., 1998, vol. 29, no. 1, pp. 927-930.

[51] J. P. Rolland, M. W. Krueger, and A. Goon, "Multifocal planes head-mounted displays," Appl. Opt., vol. 39, no. 19, pp. 3209-3215, 2000.

[52] H. Takahashi and S. Hirooka, "Stereoscopic see-through retinal projection head-mounted display," Proc. SPIE - Int. Soc. Opt. Eng., vol. 6803 , no. $1,2008,68031 \mathrm{~N}$.

[53] S. Liu and H. Hua, "Time-multiplexed dual-focal plane head-mounted display with a fast liquid lens," Opt. Lett., vol. 34, pp. 1642-1644, 2009.

[54] S. Shiwa, K. Omura, and F. Kishino, "Proposal for a 3-D display with accommodative compensation: 3DDAC,"
J. Soc. Inf. Display, vol. 4, no. 4, pp. 255-261, 1996.

[55] A. Takagi, S. Yamazaki, Y. Saito, and N. Taniguchi, "Development of a stereo video see-through HMD for AR systems," in Proc. Int. Symp. Augmented Reality, 2000, pp. 68-77.

[56] W. Robinett and J. P. Rolland, "A computational model for the stereoscopic optics of a head-mounted display," Presence, Teleoper. Virtual Environ., vol. 1, no. 1, pp. 45-62, 1992.

[57] F. L. Kooi and A. Toet, "Visual comfort of binocular and 3D displays," Displays, vol. 25, pp. 99-108, 2004.

[58] O. Cakmakci and J. Rolland, "Head-worn displays: A review," J. Display Technol., vol. 2, no. 3, pp. 199-216, Sep. 2006.

[59] G. J. Woodgate, D. Ezra, J. Harrold, N. S. Holliman, G. R. Jones, and R. R. Moseley, "Observer-tracking autostereoscopic 3D display systems," Proc. SPIE-Int. Soc. Opt. Eng., vol. 3012, no. 1, pp. 187-198, 1997.

[60] R.-Y. Tsai, C.-H. Tsai, K. Lee, C.-L. Wu, L.-C. D. Lin, K.-C. Huang, W.-L. Hsu, C.-S. Wu, C.-F. Lu, J.-C. Yang, and Y.-C. Chen, "Challenge of 3D LCD displays," Proc. SPIE-Int. Soc. Opt. Eng., vol. 7329, no. 1, 2009, 732903.

[61] M. Nishida and K. Sakamoto, "Parallax barrier 3D reflection display using polarizer slit," Proc. SPIE-Int. Soc. Opt. Eng., vol. 6392, no. 1, 2006, 63920T.

[62] C.-H. Chen, Y.-P. Huang, S.-C. Chuang, C.-L. Wu, H.-P. D. Shieh, W. Mphepö, C.-T. Hsieh, and S.-C. Hsu, "Liquid crystal panel for high efficiency barrier type autostereoscopic three-dimensional displays," Appl. Opt., vol. 48, no. 18 , pp. 3446-3454, 2009.

[63] H. Nishimura, T. Abe, H. Yamamoto, Y. Hayasaki, and N. Nishida, "Development of 140-inch autostereoscopic display by use of full-color led panel," Proc. SPIE-Int. Soc. Opt. Eng., vol. 6486, no. 1, 2007, 64861B.

[64] H. Yamamoto, M. Kouno, S. Muguruma, Y. Hayasaki, Y. Nagai, Y. Shimizu, and N. Nishida, "Enlargement of viewing area of stereoscopic full-color led display by use of a parallax barrier," Appl. Opt., vol. 41, no. 32, pp. 6907-6919, 2002

[65] K. Sakamoto and T. Morii, "Multiview 3D display using parallax barrier combined with polarizer," Proc. SPIE - Int. Soc. Opt. Eng., vol. 6399, no. 1, 2006, 63990R.

[66] D. Ezra, G. J. Woodgate, B. A. Omar, N. S. Holliman, J. Harrold, and L. S. Shapiro, "New autostereoscopic display system," Proc. SPIE-Int. Soc. Opt. Eng., vol. 2409, no. 1 , pp. 31-40, 1995.

[67] G. J. Woodgate, J. Harrold, A. M. S. Jacobs, R. R. Moseley, and D. Ezra, "Flat-panel autostereoscopic displays: Characterization and enhancement," Proc. SPIE - Int. Soc. Opt. Eng., vol. 3957, no. 1, pp. 153-164, 2000.

[68] A. Jacobs, J. Mather, R. Winlow, D. Montgomery, G. Jones, M. Willis, M. Tillin, L. Hill, M. Khazova, H. Stevenson, and G. Bourhill, "2D/3D switchable displays," Sharp Tech. J., vol. 85, pp. 15-18, 2003.

[69] D. J. Montgomery, G. J. Woodgate, A. M. S. Jacobs, J. Harrold, and D. Ezra, "Performance of a flat-panel display system convertible between 2D and autostereoscopic 3D modes," Proc. SPIE-Int. Soc. Opt. Eng., vol. 4297, no. 1, pp. 148-159, 2001. 
[70] D.-S. Kim, S. Shestak, K.-H. Cha, S.-M. Park, and S.-D. Hwang, "Time-sequential autostereoscopic OLED display with segmented scanning parallax barrier," Proc. SPIE-Int. Soc. Opt. Eng., vol. 7329, no. 1, 2009, 73290U.

[71] T. Peterka, R. L. Kooima, D. J. Sandin, A. Johnson, J. Leigh, and T. A. DeFanti "Advances in the dynallax solid-state dynamic parallax barrier autostereoscopic visualization display system," IEEE Trans. Vis. Comput. Graph., vol. 14, no. 3, pp. 487-499, May-Jun. 2008.

[72] Y.-H. Tao, Q.-H. Wang, J. Gu, W.-X. Zhao, and D.-H. Li, "Autostereoscopic three-dimensional projector based on two parallax barriers," Opt. Lett., vol. 34, no. 20, pp. 3220-3222, 2009.

[73] R. B. Johnson and G. A. Jacobsen, "Advances in lenticular lens arrays for visual display," Proc. SPIE - Int. Soc. Opt. Eng., vol. 5874, no. 1, 2005 , 587406.

[74] C.-H. Tsai, P. Lai, K. Lee, and C.-K. Lee, "Fabrication of a large f-number lenticular plate and its use as a small-angle flat-top diffuser in autostereoscopic display screens," Proc. SPIE-Int. Soc. Opt. Eng., vol. 3957, no. 1, pp. 322-329, 2000.

[75] Y.-G. Lee and J. B. Ra, "Image distortion correction for lenticular misalignment in three-dimensional lenticular displays," Opt. Eng., vol. 45, no. 1, 017007, 2006.

[76] D. K. G. de Boer, M. G. H. Hiddink, M. Sluijter, O. H. Willemsen, and S. T. de Zwart, "Switchable lenticular based 2D/3D displays," Proc. SPIE-Int. Soc. Opt. Eng., vol. 6490, 2007, 64900R.

[77] P. Benzie, J. Watson, P. Surman, I. Rakkolainen, K. Hopf, H. Urey, V. Sainov, and C. von Kopylow, "A survey of 3DTV displays: Techniques and technologies," IEEE Trans. Circuits Syst. Video Technol. vol. 17, no. 11, pp. 1647-1658, Nov. 2007.

[78] N. GmbH, NewSight 8.4" Multiview Display $A D E$, Accessed Sep. 19, 2010. [Online]. Available: http://en.souvr.com/product/p1/ p11/Index.html

[79] T. Ando, K. Mashitani, M. Higashino, H. Kanayama, H. Murata, Y. Funazou, N. Sakamoto, H. Hazama, Y. Ebara, and K. Koyamada, "Multiview image integration system for glassless 3D display," Proc. SPIE - Int. Soc. Opt. Eng., vol. 5664, no. 1, pp. 158-166, 2005.

[80] K. Mashitani, G. Hamagishi, M. Higashino, T. Ando, and S. Takemoto, "Step barrier system multiview glassless 3D display," Proc. SPIE - Int. Soc. Opt. Eng., vol. 5291, no. 1 pp. 265-272, 2004

[81] T. Endo, Y. Kajiki, T. Honda, and M. Sato, "Cylindrical 3D video display observable from all directions," Proc. SPIE-Int. Soc. Opt. Eng., vol. 3957, no. 1, pp. 225-233, 2000.

[82] H. Choi, S.-W. Min, S. Jung, J.-H. Park, and B. Lee, "Multiple-viewing-zone integral imaging using a dynamic barrier array for three-dimensional displays," Opt. Exp., vol. 11, no. 8, pp. 927-932, 2003.

[83] C. van Berkel, "Image preparation for 3D LCD," Proc. SPIE - Int. Soc. Opt. Eng., vol. 3639, no. 1, pp. 84-91, 1999.

[84] Sharp Corporation, Sharp Laboratories of Europe, Accessed Dec. 1, 2010. [Online]. http://www.sle.sharp.co.uk/ research/optical_imaging/3d_research.php
[85] Bilkent University, 3DPhone Project, Accessed Sep. 19, 2010. [Online]. Available: http://www.3dphone.org/

[86] J. C. Schultz, R. Brott, M. Sykora, W. Bryan, T. Fukamib, K. Nakao, and A. Takimoto, "11.5l: Late-news paper: Full resolution autostereoscopic 3d display for mobile applications," in Dig. Tech. Papers SID Symp., 2009, vol. 40, no. 1, pp. 127-130.

[87] A. Travis, N. Emerton, T. Large, S. Bathiche, and B. Rihn, "Backlight for view-sequential autostereo 3D," in Dig. Tech. Papers SID Symp., 2010, pp. 215-217.

[88] H. Baker and Z. Li, "Camera and projector arrays for immersive 3D video," in Proc. 2nd Int. Conf. Immersive Telecommun., Brussels, Belgium, 2009, pp. 1-6.

[89] J. Flack, J. Harrold, and G. J. Woodgate, "A prototype 3D mobile phone equipped with a next-generation autostereoscopic display," Proc. SPIE - Int. Soc. Opt. Eng., vol. 6490, no. 1, 2007, 64900M

[90] D. Ezra, G. J. Woodgate, B. A. Omar, N. S. Holliman, J. Harrold, and L. S. Shapiro, "New autostereoscopic display system," Proc. SPIE-Int. Soc. Opt. Eng., vol. 2409, no. 1, pp. 31-40, 1995.

[91] T. Hattori, Sea Phone 3D Display, Accessed Mar. 15, 2010. [Online]. Available: http:// home.att.net/SeaPhone/3display.htm

[92] S. A. Benton, "Autostereoscopic display system," U.S. Patent 6351 280, 2002.

[93] J.-Y. Son, S. A. Shestak, S.-S. Kim, and Y.-J. Choi, "Desktop autostereoscopic display with head tracking capability," Proc. SPIE-Int. Soc. Opt. Eng., vol. 4297, no. 1, pp. 160-164, 2001.

[94] S. Ichinose, N. Tetsutani, and M. Ishibashi, "Full-color stereoscopic video pickup and display technique without special glasses," in Dig. Tech. Papers SID Symp., 1989, vol. 3014 no. 4, pp. 319-324.

[95] T. Okoshi, Three Dimensional Imaging Techniques. New York: Academic, 1976.

[96] N. Tetsutani, S. Ichinose, and M. Ishibashi, "3D-TV projection display system with head tracking," in Proc. Jpn. Display, 1989, pp. 56-59.

[97] P. V. Harman, "Autostereoscopic teleconferencing system," Proc. SPIE-Int Soc. Opt. Eng., vol. 3957, no. 1, pp. 293-302, 2000.

[98] C. Hembd, R. Stevens, and M. Hutley, "Imaging properties of the gabor superlens," in Eur. Opt. Soc., Topical Meetings Dig. 13 Microlens Arrays, May 15-16, 1997, pp. 101-104.

[99] N. Tetsutani, K. Omura, and F. Kishino, "Study on a stereoscopic display system employing eye-position tracking for multi-viewers," Proc. SPIE-Int. Soc. Opt. Eng., vol. 2177, no. 1, pp. 135-142, 1994.

[100] A. Arimoto, T. Ooshima, T. Tani, and Y. Kaneko, "Wide-viewing-area glassless stereoscopic display using multiple projectors," Proc. SPIE-Int. Soc. Opt. Eng., vol. 3295, no. 1, pp. 186-192, 1998

[101] A. Schwartz, "Head tracking stereoscopic display," in Proc. IEEE Int. Display Res. Conf., 1985, pp. 141-144.

[102] T. Hattori, S. Sakuma, K. Katayama, S. Omori, M. Hayashi, and M. Yokoi, "Stereoscopic liquid crystal display i (general description)," Proc. SPIE-Int. Soc. Opt. Eng., vol. 2177, no. 1, pp. 143-149, 1994.
103] D. J. Sandin, T. Margolis, G. Dawe, J. Leigh, and T. A. DeFanti, "Varrier autostereographic display Proc. SPIE-Int. Soc. Opt. Eng., vol. 4297, no. 1, pp. 204-211, 2001.

[104] K. Perlin, C. Poultney, J. S. Kollin, D. T. Kristjansson, and S. Paxia "Recent advances in the NYU autostereoscopic display," Proc. SPIE-Int. Soc. Opt. Eng., vol. 4297, no. 1, pp. 196-203, 2001.

[105] A. Schwerdtner and H. Heidrich, "Dresden 3D display (D4D)," Proc SPIE-Int. Soc. Opt. Eng., vol. 3295, pp. 203-210, Apr. 1998

[106] D. J. Trayner and E. Orr, "Developments in autostereoscopic displays using holographic optical elements," Proc. SPIE-Int. Soc. Opt. Eng., vol. 3012, no. 1, pp. 167-174, 1997.

[107] Heinrich Hertz Institute, Free2C Desktop Display, Accessed Mar. 15, 2010. [Online]. Available: http://www.hhi.fraunhofer.de/ en/departments/interactive-media-humanfactors/overview/free2c-desktop-display/

[108] S. GmbH, SeeFront 3D Displays, Accessed Mar. 15, 2010. [Online]. Available: http:// www.seefront.com/

[109] R. Häussler, A. Schwerdtner, and N. Leister, "Large holographic displays as an alternative to stereoscopic displays," Proc. SPIE - Int. Soc. Opt. Eng., vol. 6803, no. 1, 2008 , $68030 \mathrm{M}$

[110] P. Surman, I. Sexton, K. Hopf, W. K. Lee, F. Neumann, E. Buckley, G. Jones, A. Corbett, R. Bates, and S. Talukdar, "Laser-based multi-user 3-d display," J. Soc. Inf. Display, vol. 16, pp. 743-753, 2008.

[111] V. C. Kishore, E. Erden, H. Urey, H. Baghsiahi, E. Willman, S. E. Day, D. R. Selviah, F. A. Fernández, and P. Surman, "Laser scanning 3D display with dynamic exit pupil," in Proc. 29th Int Display Res. Conf., 2009, pp. 492-495.

[112] P. Surman, I. Sexton, K. Hopf, W. K. Lee, E. Buckley, G. Jones, and R. Bates, "European research into head tracked autostereoscopic displays," in Proc. 3DTV Conf., 2008, pp. 161-164.

[113] E. Erden, V. C. Kishore, H. Ürey, H. Baghsiahi, E. Willman, S. E. Day, D. R. Selviah, F. A. Fernandez, and P. Surman, "Laser scanning based autostereoscopic 3D display with pupil tracking," in Proc. IEEE Photonics, 2009, pp. 10-11.

[114] S. Pastoor, "Human factors of 3DTV: An overview of current research at Heinrich-Hertz-Institut Berlin," in IEE Colloq. Stereoscopic Television, Oct. 1992, pp. 11/1-11/4.

[115] Y. Takaki, "High-density directional display for generating natural three-dimensional images," Proc. IEEE, vol. 94, no. 3, pp. 654-663, Mar. 2006.

[116] Sunny Ocean Studios, 3D Displays, Accessed Mar. 15, 2010. [Online]. Available: http:// www.sunny-ocean.com/displays.html

[117] T. Baloch, "Method and apparatus for displaying three-dimensional images," U.S. Patent 6201565 B1, 2001.

[118] C. Cameron, Collaborative Autostereo 3D Display System, Accessed Mar. 15, 2010. [Online]. Available: http://www.sid.org/ chapters/uki/presentations/qinetiq.pdf

[119] M. Sayinta and H. Urey, "Scanning LED array based volumetric display," in Proc. 3DTV Conf., May 2007, pp. 1-4. 
[120] Holografika, Real 3D Display, Accessed Mar. 15, 2010. [Online]. Available: http://www.holografika.com/

[121] H. Urey, S. Holmstrom, and A. D. Yalcinkaya, "Electromagnetically actuated FR4 scanners," IEEE Photon. Technol. Lett., vol. 20, no. 1, pp. 30-32, Jan. 2008.

[122] H. Tilton, "Large-CRT holoform display," in Proc. IEEE Int. Display Res. Conf., 1985, pp. 145-146.

[123] C. Moller and A. R. L. Travis, "Flat panel time multiplexed autostereoscopic display using an optical wedge waveguide," in Proc. 11th Int. Display Workshop, 2004 pp. 1443-1446.
[124] Y. Kajiki, H. Yoshikawa, and T. Honda, "Hologram-like video images by 45-view stereoscopic display," Proc. SPIE - Int. Soc. Opt. Eng., vol. 3012, no. 1, pp. 154-166, 1997.

[125] M. Okui, J. Arai, and F. Okano, New Integral Imaging Technique Uses Projector, SPIE News Room, 2007, pp. 1-3.

[126] Hitachi Shows 10" Glasses-Free 3D Display, Accessed 8 Sept. 2010. [Online]. Available: http://www.3d-display-info.com/hitachishows-10-glasses-free-3d-display

[127] H. Baker and Z. Li, "Camera and projector arrays for immersive 3D video," in Proc. 2nd Int. Conf. Immersive Telecommun., Brussels, Belgium, 2009, pp. 1-6.
[128] H. Urey and K. D. Powell, "Microlens-array-based exit-pupil expander for full-color displays," Appl. Opt., vol. 44, pp. 4930-4936, 2005

[129] A. J. Woods and T. Rourke, "Ghosting in anaglyphic stereoscopic images," in Proc. Conf. Stereoscopic Displays Virtual Reality Syst., San Jose, CA, Jan. 19-22, 2004.

[130] I. Ideses and L. Yaroslavsky, "New methods to produce high quality color anaglyphs for 3-D visualization," in Proc. Int. Conf. Image Anal. Recognit., Porto, Portugal, Sep. 29-Oct. 1, 2004

\section{ABOUT THE AUTHORS}

Hakan Urey (Senior Member, IEEE) received the B.S. degree from the Middle East Technical University, Ankara, Turkey, in 1992 and the M.S. and Ph.D. degrees from Georgia Institute of Technology, Atlanta, in 1996 and 1997, respectively, all in electrical engineering.

Currently, he is a Professor of Electrical Engineering at Koç University, Istanbul, Turkey. After completing his Ph.D., he joined Microvision Inc., Seattle, WA, as a Research Engineer and he played

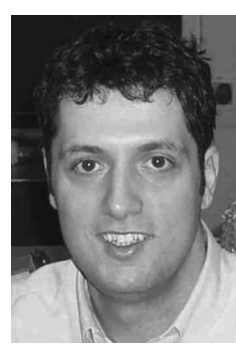
a key role in the development of the laser scanning display technologies. He was the Principal System Engineer when he left Microvision to join the faculty of engineering at Koç University, where he established the Optical Microsystems Research Laboratory (OML). He published 35 journal and about 80 conference papers, seven edited books, two book chapters, and he has 20 issued and several pending patents. His research interests are in optical microelectromechanical systems (MEMS), optical system design, and laser-based 2-D/3-D display and imaging systems, and biosensors.

Dr. Urey received the Werner Von Siemens Faculty Excellence Award in 2006 from Koç University, the Distinguished Young Scientist award from the Turkish Academy of Sciences in 2007, and the Encouragement Award from the Scientific and Technical Research Council of Turkey (TUBITAK) in 2009. He is a member of The International Society for Optical Engineers (SPIE), the Optical Society of America (OSA), IEEE-Photonics, and the Vice President of the Turkey chapter of IEEE-Photonics.

Kishore V. Chellappan received the M.Sc. and Ph.D. degrees in physics from Cochin University of Science and Technology, Kerala, India, in 2002 and 2009, respectively.

He joined the Optical Microsystems Laboratory, Koç University, Istanbul, Turkey, in 2008, where he contributed to the research and development efforts of the EC funded "HELIUM3D" laser-based display project. He is now a Research Associate at the University of Southern

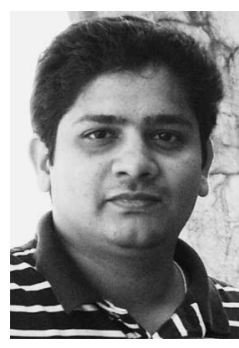
Mississippi, Hattiesburg. His research interests are in laser-based displays, optical system design, photorefractive polymers, photopolymers, and infrared spectroscopy. He is a coauthor of several publications including one book chapter.

Dr. Chellappan is a member of The International Society for Optical Engineers (SPIE) and the Optical Society of America (OSA).
Erdem Erden (Student Member, IEEE) received the B.Sc. degree in electrical and electronics engineering and the M.Sc. degree in electrical and computer engineering from Koç University, Istanbul, Turkey, in 2008 and 2010, respectively. Currently, he is working towards the Ph.D. degree at the Center for Research and Education in Optics and Lasers (CREOL), University of Central Florida, Orlando.

He has worked at Optical Microsystems Labo-

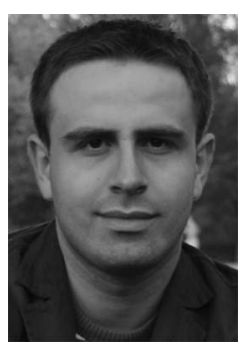
ratory at Koç University as a Research Assistant between 2007 and 2010 and he has recently joined the Optical Fiber Communications Laboratory at CREOL as a Graduate Research Assistant. His research interests are 3-D displays, biophotonics, optical fiber telecommunication, and optical microelectromechanical systems (MEMS).

Mr. Erden received the Vehbi Koç Foundation scholarship and the Scientific and Technological Research Council of Turkey (TUBITAK) scholarship during his studies.
Phil Surman received the B.Sc degree in electrical and electronic engineering from Heriot-Watt University, Edinburgh, U.K., in 1971 and the Ph.D. degree in the subject of head-tracked 3-D displays from De Montfort University, Leicester, U.K., in 2003.

He has been investigating 3-D displays for the last 23 years and holds the patents on the optical systems for the MUTED and HELIUM3D displays. $\mathrm{He}$ is currently a Senior Research Fellow in the

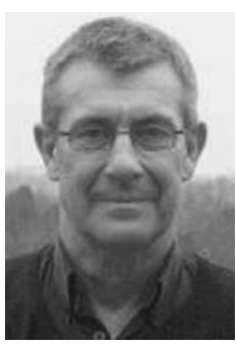
Imaging and Displays Research Group at De Montfort University and has been involved in the European Union-funded ATTEST, 3D TV Network of Excellence, MUTED and HELIUM3D projects that are all in the area of 3-D displays. 